\title{
Pazopanib Sensitivity in a Patient With Breast Cancer and FGFR1 Amplification
}

\author{
Fiona Tsui-Fen Cheng, MD, MS,a,; Fu Ou-Yang, MD, PhD ${ }^{\text {b,c,*; }}$;ina Lapke, Dr. rer. nat. ${ }^{\text {d; }}$; \\ Kai-Che Tung, MS ${ }^{\mathrm{d}, \mathrm{e}}$; Yen-Kung Chen, MD, PhD; Yuh-Yu Chou, MD;; and Shu-Jen Chen, $\mathrm{PhD}^{\mathrm{d}}$
}

\begin{abstract}
Treatment options for patients with hormone receptor-positive (HR+), HER2-negative (HER2-) breast cancer and resistance to endocrine therapy remain limited. An interesting therapeutic target in these patients is fibroblast growth factor receptor 1 (FGFR1). FGFR1 is amplified in approximately $11 \%$ of patients with breast cancer, especially those with HR+ disease. This report presents a patient with metastatic HR+ HER2- breast cancer harboring an FGFR1 amplification who was resistant to endocrine therapy but responded to pazopanib, a multityrosine kinase inhibitor with FGFR-inhibiting activity. Upon pazopanib treatment, the patient's brain lesions nearly disappeared, and she experienced therapeutic changes in the lung and an improvement of liver function. This case suggests that pazopanib may be a promising agent for the treatment of patients with breast cancer and FGFR1 amplifications.
\end{abstract}

J Natl Compr Canc Netw 2017;15(12):1456-1459 doi: 10.6004/jncen.2017.7030

\section{Case Report}

A 47-year-old Asian woman presented at our institution with stage IIIA (T1cN2M0) invasive ductal carcinoma. She underwent breast-conserving surgery and axillary lymph node dissection. Her tumor was found to be grade II, estrogen receptor-positive, progesterone receptor-negative, and HER2-negative (HER2-), and had spread to 10 of 17 dissected lymph nodes. The patient was treated with TEC (taxotere, epirubicin, and cyclophosphamide) adjuvant chemotherapy for 3.5 months followed by endocrine therapy with goserelin and tamoxifen. This regimen was continued for 2 years until the patient experienced relapse with multiple lung metastases. She received various therapies over the next 4.5 years, including chemotherapy, endocrine therapy, radiotherapy, and targeted therapy. The chemothera-

From the aBreast Cancer Center, Shin Kong Wu Ho-Su Memorial Hospital,

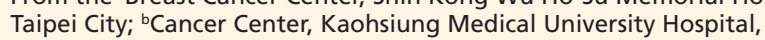

Kaohsiung; 'Kaohsiung Medical University, Kaohsiung; ${ }^{\mathrm{d}} \mathrm{ACT}$ Genomics

Co., LTD, Taipei City; 'Department of Medical Science and Institute of

Bioinformatics and Structural Biology, National Tsing Hua University,

Hsinchu; and Departments of fNuclear Medicine and 'Pathology, Shin Kong

Wu Ho-Su Memorial Hospital, Taipei City, Taiwan.

*These authors contributed equally.

Submitted April 24, 2017; accepted for publication September 5, 2017.

Drs. Lapke, Tung, and S.J. Chen are employees of ACT Genomics. Drs. peutic drugs used were vinorelbine, gemcitabine, UFUR (tegafur/uracil), CMF (cyclophosphamide, methotrexate, and fluorouracil), eribulin, capecitabine, and paclitaxel, and the endocrine drugs were goserelin, fulvestrant, letrozole, and exemestane; everolimus was used for targeted therapy.

However, the patient's disease progressed, and she was diagnosed with brain metastasis after having a seizure. To determine suitable treatment options, she underwent comprehensive tumor genetic testing via nextgeneration sequencing of all coding exons of $>400$ cancer-related genes (ACTOnco, ACT Genomics Co., LTD, Taipei City, Taiwan) to detect sequence variants and copy number alterations, as well as testing for genetic alterations of BRCA1 and BRCA2 (ACTBRCA, ACT Genomics Co., LTD). The tests were performed

Lapke and S.J. Chen own stock in ACT Genomics. The remaining authors have disclosed that they have no financial interests, arrangements, affiliations, or commercial interests with the manufacturers of any products discussed in this article or their competitors.

Correspondence: Nina Lapke, Dr. rer. nat., ACT Genomics Co., LTD, 1F., No. 280, Xinhu 2nd Road, Neihu District, Taipei City 114, Taiwan (R.O.C.). E-mail: ninalapke@actgenomics.com

Shu-Jen Chen, PhD, ACT Genomics Co., LTD, 3F., No. 345, Xinhu 2nd Road, Neihu District, Taipei City 114, Taiwan (R.O.C.).

E-mail: sjchen@actgenomics.com 
on an archival formalin-fixed, paraffin-embedded sample of a neck lymph node isolated 2.5 years earlier. Results revealed an unstable copy number profile of the tumor (Figure 1) with copy number loss on chromosome 13, which resulted in a loss of one copy of BRCA2 and a loss of both copies of RB1. The RB1 loss indicated that the patient would be unlikely to benefit from palbociclib treatment. However, an amplification was observed for chromosome 8 , including the gene for fibroblast growth factor receptor 1 (FGFR1) with an observed copy number of 9. Importantly, no activating mutations in FGFR1 downstream signaling pathway genes, such as KRAS, were detected. These results indicated that the patient could benefit from treatment with FGFR inhibitors, such as pazopanib.

The patient received pazopanib treatment for 7.5 months at an oral dose of 400 to $600 \mathrm{mg} / \mathrm{d}$. The major adverse effect of pazopanib treatment was fatigue. After 3 months of treatment, therapeutic changes were evaluated by radiologic imaging. The most striking treatment effect was observed in the brain. Before treatment, tumors with internal calcifications and perifocal edema were observed in the left cerebral hemisphere (Figure 2A); after 3 months of treatment, metastases were nearly absent and there was a decrease of perifocal edema. Those changes represent a partial response according to RECIST 1.1 criteria. In addition, therapeutic changes were seen in the lung metastases (Figure 2B), and levels of the tumor markers carcinoembryonic antigen and CA-153 decreased (Figure 3A and B). An im- provement of liver-related disease was also seen, as indicated by decreased serum bilirubin levels (Figure 3C). Furthermore, the patient's jaundice and clay stools improved after 3 weeks of treatment, and did not worsen thereafter. Unfortunately, the patient eventually experienced relapse and died 7.5 months after pazopanib initiation.

\section{Discussion}

This case report suggests that pazopanib treatment may be a promising option for patients with metastatic hormone receptor-positive $(\mathrm{HR}+)$ breast cancer with FGFR1 amplifications for whom previous lines of treatment have failed. Patients with HR+ breast cancer are sensitive to endocrine therapy and have a considerably better prognosis than those with HR-negative disease. ${ }^{1}$ However, resistance to endocrine therapy may develop, presenting a need to find alternative treatment options. To find a matching, personalized targeted therapy for these patients, genetic profiling may be used to identify relevant oncogenic drivers.

One such driver is FGFR1. Amplifications of FGFR1 are correlated with enhanced protein expression and deliver important growth and survival signals to tumor cells, and overexpression of FGFR1 is associated with a shorter distant metastasis-free survival. ${ }^{2}$ FGFR1 amplification occurs in approximately $11 \%$ of patients with breast cancer, especially those with HR+ tumors. ${ }^{3,4}$ Preclinical data indicate that FGFR1 amplifications may be involved in mediating

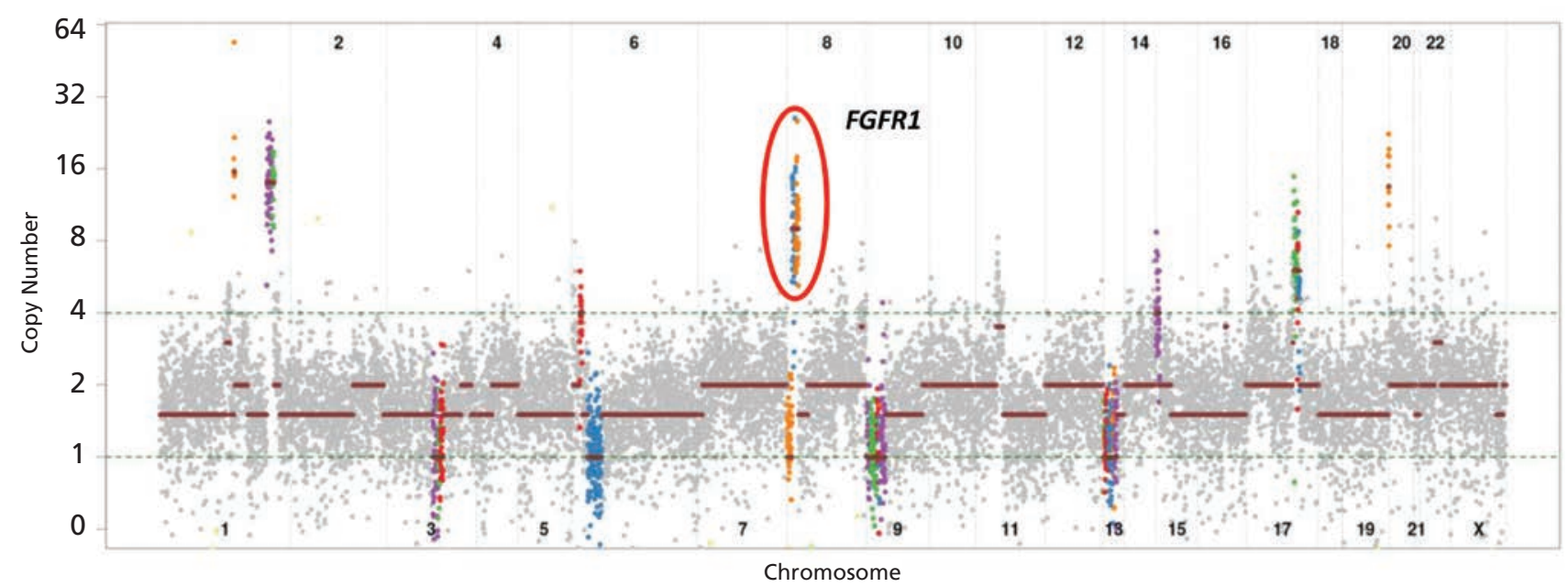

Figure 1. Copy number profile of the patient's neck lymph node formalin-fixed, paraffin-embedded tumor sample. Amplified regions of chromosome 8 are indicated by a red circle and include the gene encoding fibroblast growth factor receptor 1 (FGFR1). 
Cheng et al

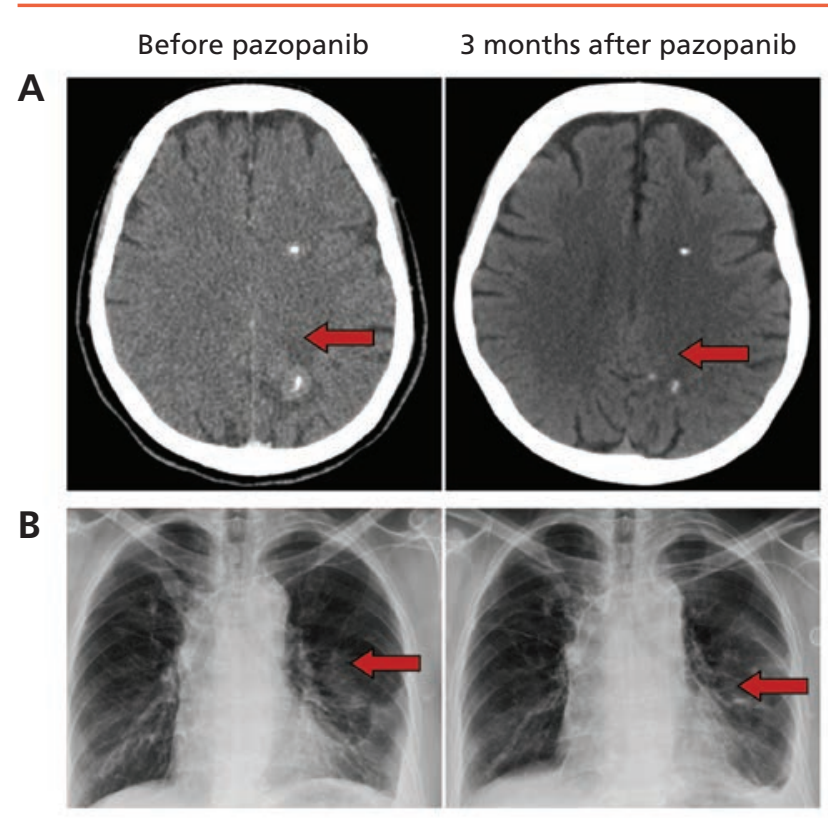

Figure 2. Shrinkage of tumor lesions prior to initiation and 3 months after starting pazopanib treatment for (A) brain and (B) lung. Lesions are indicated by red arrows.

resistance to endocrine therapy. ${ }^{2}$ This is in line with the observation that therapy resistance is particularly common in patients with luminal B tumors, ${ }^{5}$ among whom $16 \%$ to $27 \%$ harbor the alteration. ${ }^{2}$ Preclinical data further indicate that FGFR inhibitors may be effective in patients with FGFR amplification, ${ }^{6,7}$ and some available clinical data support this notion. Among patients with breast cancer treated with the experimental FGFR inhibitor dovitinib, those with FGFR1 amplifications and a copy number $\geq 6$ seemed to benefit more from treatment. ${ }^{8}$ Similarly, among 8 patients with breast cancer and FGFR1 amplifications who were treated with AZD4547, only the patient with the highest copy number experienced a response. ${ }^{9}$ However, not all studies found an association between a high FGFR1 copy number and response to FGFR inhibition, ${ }^{10}$ and further studies are required.

An FDA-approved FGFR inhibitory agent is the multi-tyrosine kinase inhibitor (TKI) pazopanib, which is indicated for the treatment of renal cell carcinoma and soft tissue sarcoma. This case report shows that pazopanib induced an encouraging clinical response in a patient with breast cancer and FGFR1 amplification. Importantly, the patient was heavily pretreated and had metastases in the liver, lung, and brain. Upon treatment, the brain lesions nearly disappeared, and therapeutic changes in the lung, an improvement of liver function, and

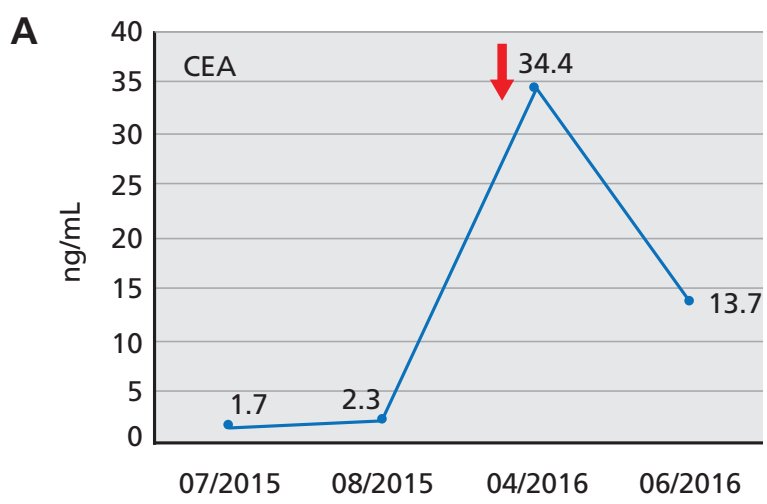

B

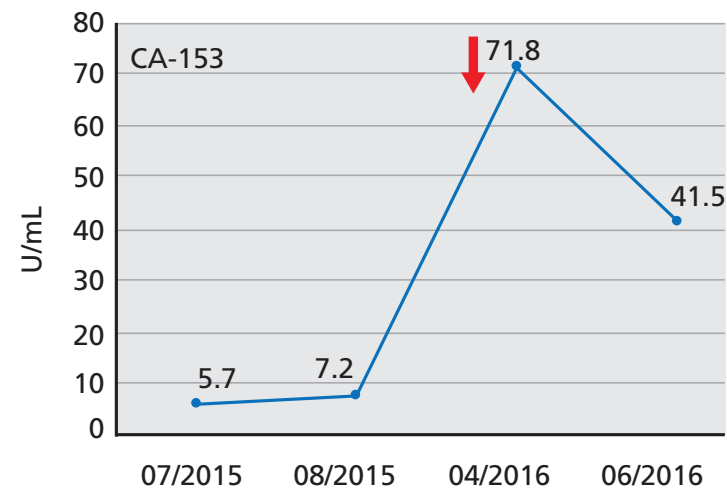

C

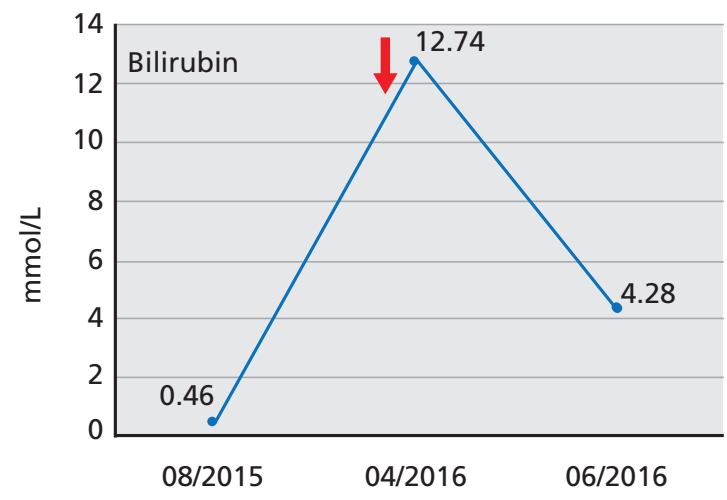

Figure 3. Change in levels of blood biomarkers upon pazopanib treatment for (A) carcinoembryonic antigen (CEA), (B) CA-153, and (C) bilirubin. Pazopanib treatment was initiated on March 14, 2016, as indicated by red arrows.

a decrease in tumor markers occurred. Unfortunately, the patient experienced relapse and died 7.5 months after pazopanib initiation. However, the clinical improvement observed for this patient represents an impressive treatment success considering the patient's disease presentation before treatment.

Patients with $\mathrm{HR}+$, endocrine therapy-resistant breast cancer represent a considerably large subgroup. This subgroup has previously been shown to 
benefit from pazopanib treatment, ${ }^{11}$ but biomarkers for response to this agent need to be identified, also because of toxicity concerns. ${ }^{12}$ A promising biomarker for pazopanib and other FGFR inhibitors may be FGFR1 amplification, and in addition to the present case report, another recent publication reports stable disease in a patient with $\mathrm{HR}+$ breast cancer with FGFR1 amplification. ${ }^{13}$ Further potential markers for pazopanib treatment include alterations in other FGFR or FGFR ligand genes. Future studies may be needed to further investigate biomarker validity and determine cutoffs for different agents. ${ }^{8-10}$

Because pazopanib is a multi-TKI with vascular endothelial growth factor receptor (VEGFR) inhibitory properties, VEGFR inhibition may have contributed to the tumor shrinkage observed in our patient. Studies with different FGFR inhibitory agents enrolling higher numbers of patients with FGFR1 amplifications may shed light on the effectiveness of multi-TKIs with broad activity compared with more specific FGFR inhibitors, such as AZD4547.

Our case report highlights the value of comprehensive genomic profiling by next-generation sequencing to identify therapeutic options for patients with endocrine therapy-resistant breast cancer in a personalized way. By sequencing $>400$ cancer-related genes, we were not only able to detect the FGFR1 amplification, but could also ensure that important genes downstream of FGFR1, such as KRAS, ${ }^{2}$ were unaltered. This made a response to FGFR inhibition more likely, not just from a single-gene but also from a whole-pathway perspective. It would be interesting to know whether the presence of downstream mutations may have contributed to the resistance of patients with FGFR1 amplifications on treatment with the FGFR inhibitors dovitinib ${ }^{8,10}$ or AZD4547. ${ }^{9}$ We further detected a deletion of both copies of RB1, indicating that the patient would likely not have benefitted from palbociclib. ${ }^{14}$

\section{Conclusions}

This case report identifies pazopanib as a targeted agent that may be considered for the treatment of patients with $\mathrm{HR}+$, endocrine therapy-resistant metastatic breast cancer who harbor FGFR1 amplifications. Future studies are warranted to further evaluate this treatment option.

\section{References}

1. Viale G, Regan MM, Maiorano E, et al. Prognostic and predictive value of centrally reviewed expression of estrogen and progesterone receptors in a randomized trial comparing letrozole and tamoxifen adjuvant therapy for postmenopausal early breast cancer: BIG 1-98. J Clin Oncol 2007;25:3846-3852.

2. Turner N, Pearson A, Sharpe R, et al. FGFR1 amplification drives endocrine therapy resistance and is a therapeutic target in breast cancer. Cancer Res 2010;70:2085-2094.

3. Courjal F, Cuny M, Simony-Lafontaine J, et al. Mapping of DNA amplifications at 15 chromosomal localizations in 1875 breast tumors: definition of phenotypic groups. Cancer Res 1997;57:4360-4367.

4. Cancer Genome Atlas Network. Comprehensive molecular portraits of human breast tumours. Nature 2012;490:61-70.

5. Ades F, Zardavas D, Bozovic-Spasojevic I, et al. Luminal B breast cancer: molecular characterization, clinical management, and future perspectives. J Clin Oncol 2014;32:2794-2803.

6. Shiang CY, Qi Y, Wang B, et al. Amplification of fibroblast growth factor receptor- 1 in breast cancer and the effects of brivanib alaninate. Breast Cancer Res Treat 2010;123:747-755.

7. Gozgit JM, Wong MJ, Moran L, et al. Ponatinib (AP24534), a multitargeted pan-FGFR inhibitor with activity in multiple FGFR-amplified or mutated cancer models. Mol Cancer Ther 2012;11:690-699.

8. Andre F, Bachelot $\mathrm{T}$, Campone $\mathrm{M}$, et al. Targeting FGFR with dovitinib (TKI258): preclinical and clinical data in breast cancer. Clin Cancer Res 2013;19:3693-3702.

9. Pearson A, Smyth E, Babina IS, et al. High-level clonal FGFR amplification and response to FGFR inhibition in a translational clinical trial. Cancer Discov 2016;6:838-851.

10. Musolino A, Campone M, Neven P, et al. Phase II, randomized, placebo-controlled study of dovitinib in combination with fulvestrant in postmenopausal patients with HR+, HER2- breast cancer that had progressed during or after prior endocrine therapy. Breast Cancer Res 2017;19:18

11. Majure MC, Melisko ME, Karwat AR, et al. Role of pazopanib (PZ) in modulating hormone resistance in advanced breast cancer $(\mathrm{ABC})$ [abstract]. J Clin Oncol 2015;33(Suppl):Abstract 529.

12. Tan AR, Johannes $H$, Rastogi $P$, et al. Weekly paclitaxel and concurrent pazopanib following doxorubicin and cyclophosphamide as neoadjuvant therapy for HER-negative locally advanced breast cancer: NSABP Foundation FB-6, a phase II study. Breast Cancer Res Treat 2015;149:163169.

13. Yuan $Y$, Yost SE, Yuan YC, et al. Genomic mutation-driven metastatic breast cancer therapy: a single center experience. Oncotarget 2017;8:26414-26423.

14. Herrera-Abreu MT, Palafox M, Asghar U, et al. Early adaptation and acquired resistance to CDK4/6 inhibition in estrogen receptor-positive breast cancer. Cancer Res 2016;76:2301-2313. 